\title{
NGC 3293 revisited by the Gaia-ESO Survey
}

\author{
Thierry Semaan ${ }^{1}$, Thierry Morel ${ }^{1}$, Eric Gosset ${ }^{1}$, Juan Zorec $^{2}$, \\ Yves Frémat ${ }^{3}$, Ronny Blomme ${ }^{3}$ and Alex Lobel ${ }^{3}$ \\ ${ }^{1} \mathrm{ULg}$, Institut d'Astrophysique et de Géophysique, Liège, Belgium \\ email: thierry.semaan@ulg.ac.be \\ ${ }^{2}$ Institut d'Astrophysique de Paris, CNRS (UMR 7095), Paris, France \& \\ Université Pierre et Marie Curie (UMR 7095), Paris, France \\ ${ }^{3}$ Royal Observatory of Belgium, Brussels, Belgium
}

\begin{abstract}
In the framework of the Gaia-ESO survey we have determined the fundamental parameters of a large number of B-type stars in the Galactic, young open cluster NGC 3293. The determination of the stellar parameters is based on medium-resolution spectra obtained with FLAMES/GIRAFFE at ESO-VLT. As a second step, we adopted the accurate parameters to determine the chemical abundances of these hot stars. We present a comparison of our results with those obtained by the 'VLT-FLAMES survey of massive stars' (Evans et al. 2005). Our study increases the number of objects analysed and provides an extended view of this cluster.
\end{abstract}

Keywords. Massive Stars, Fundamental parameters, Chemical abundances

\section{Observations, reduction and stars analysed}

The observations of NGC 3293 with the multifiber spectrograph FLAMES/GIRAFFE took place from mid-February until beginning of April 2012. During this period, 25 FLAMES/GIRAFFE frames were obtained in the Medusa mode at medium resolution. The GIRAFFE observations were made in 4 grating setups: HR3, HR5A, HR6, and HR14A, which correspond to the wavelength-intervals 4030-4200, $4350-4750 \AA$ and 6300-6700 A respectively, with a mean resolving power of 20000. The HR3, HR5A, and HR6 domains include several strong helium lines, as well as weak SiII, SirII, CII and OII lines that are very useful for the determination of the fundamental parameters of hot stars. The HR14A domain contains $\mathrm{H} \alpha$ and can be used to identify the emission-line stars. The reduction of the GIRAFFE spectra was done by the Cambridge Astronomy Survey Unit (CASU) using a dedicated pipeline. Thanks to the 25 GIRAFFE frames we have more than 500 stars totally covered by the 4 setups. We select only the B stars (no $\mathrm{O}$ stars are present in this dataset) thanks to a visual inspection. In a second step we normalised these spectra to the continuum flux level. In our sample we have also detected Be stars, as well as single (SB1) and double-lined binary systems (SB2). The objects firmly identified as Be stars or SB2 systems were excluded from the final sample.

\section{Determination of the fundamental parameters}

The fundamental parameters $\left(T_{\text {eff }}, v \sin i, \log g\right.$ ) and the radial velocities of the $\mathrm{B}$ stars are obtained using the GIRFIT code (Frémat et al. 2006). This program is based on a least square method and derives the parameters by fitting the observed, normalised spectra with a grid of stellar fluxes computed with the atmospheric code TLUSTY in nonlocal thermodynamical (NLTE) equilibrium mode. The determination of the fundamental parameters is performed over the whole wavelength domain of 4030-4200 $\AA$ and 4350 $4750 \AA$ with exceptions for the interstellar bands and the emission components that 


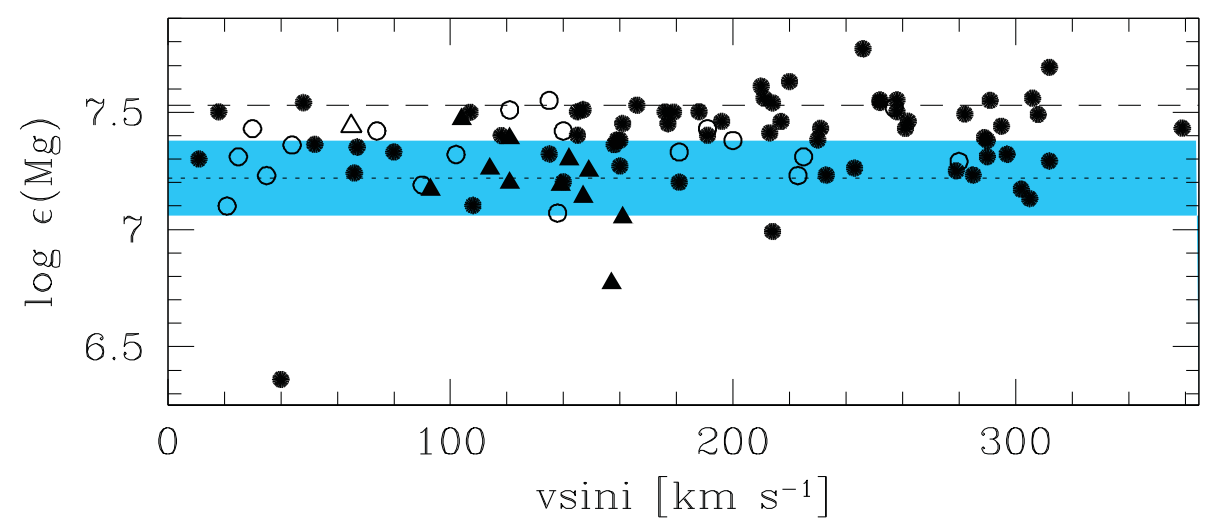

Figure 1. Variation of the $\mathrm{Mg}$ abundance, as a function of $v \sin i$. The open symbols represent possible SB2 and all SB1 systems. The horizontal blue strip shows the mean value for this cluster determined by Hunter et al. (2009). The horizontal dashed line shows the meteoritic abundance (Asplund et al. 2009).

sometimes are filling in the Balmer lines. The microturbulence velocity is fixed in the fitting procedure, but we have computed three different grids for 2,5 and $10 \mathrm{~km} \mathrm{~s}^{-1}$. This allows us to determine the best microturbulence velocity among the 3 values for each star.

\section{Determination of chemical abundances}

After determining $T_{\mathrm{eff}}, \log g, v \sin i$ and the best microturbulence velocity, we use these parameters to compute the abundances of $\mathrm{He}, \mathrm{Mg}$ and $\mathrm{Si}$. They are obtained through a best match search between a grid of synthetic spectra and observed line profiles of HeI 4471, HeI 4713, MgII 4481, SirII 4568, and SirII 4575. The grid of synthetic spectra is generated by the NLTE code DETAIL/SURFACE. The variation of the Mg abundance as a function of $v \sin i$ is shown in Fig. 1. Our values are slightly larger than those obtained by Hunter et al. (2009) and no dependence on $T_{\text {eff }}, \log g$ or $v \sin i$ is detected.

\section{Conclusion}

This work must be completed with the UVES spectra, which have not yet been analysed. Indeed, this includes some bright stars whose analysis will certainly complete and improve our results. The abundances of some key elements (e.g., CNO) will be determined for the hottest stars of the cluster such that the mixing processes could then be investigated. In the same way, the analysis of Trumpler 14 and NGC 6705 will be completed in the near future.

\section{References}

Asplund, M., Grevesse, N., Sauval, A. J., \& Scott, P. 2009, ARA\& A 47, 481

Evans, C. J., Smartt, S. J., Lee, J.-K., et al. 2005, A\&A 437, 467

Frémat, Y., Neiner, C., Hubert, A.-M., et al. 2006, A\&\&A 451, 1053

Hunter, I., Brott, I., Langer, N., et al. 2009, A\&A 496, 841 